\title{
Presidential-Lecture
}

\section{Setting up a division of HBP surgery at a local university hospital}

\author{
Yang Won $\mathrm{NAH}^{*}$ \\ Department of Surgery, Ulsan University Hospital, University of Ulsan College of Medicine, Ulsan, Korea
}

Lecture: Dear members and colleagues, it is my great honor being the president of our society, Korean Association of HBP Surgery over the last year and being here to present my talk today as well.

Ulsan University Hospital (UUH) was designated as a university hospital in March 1997 but was not gaining the trust of the local people at the time. It had been a general hospital and not specialized in the care of malignancy or organ transplantation. When I was appointed to the UUH in 2001, I thought about how to gain the trust of the local people. If there is a reliable hospital in the community, it would be helpful to the local people themselves.

Liver transplant surgery is an operation with extreme results in life and death. So I thought that if we get good results from liver transplantation, that will lead to the trust of the local people. And I believed it would be mutual benefit. We started to build up a liver transplant program at UUH. Besides HBP surgery team, clinicians including hepatologist, radiologist, anesthesiologist, interventionist, clinical pathologist and internist of infectious disease et cetera made their best efforts to build up the liver transplantation (LT) program at their respective positions. Nursing teams working at the operating room and intensive care unit were sent to the Asan Medical Center for professional training several times. After nearly one year of preparation, the first liver transplant surgery was successfully performed in March 2002 with the aid of my mentor professor SG Lee.

My interest in liver transplantation goes back to the days of my tenure at Inje University Seoul Paik Hospital, where I had trained under my first mentor professor Hyucksang Lee from basic surgical skills. I had a fortunate to implement a nation-wide survey of surgical patients with hepatocellular carcinoma (HCC), intrahepatic cholangiocarcinoma as well as liver transplantation under the direction of professor HS Lee. It's awkward to say, but until mid-1990's, the survival rate of patients after liver transplantation in Korea was dismal, and LT programs were often given up soon after they were created. According to the most recent data, Korean Network for Organ Sharing (KNOS) reported 11 year patient survival rate after LT as $71.6 \%$. This was not inferior to the United Network for Organ Sharing (UNOS) result of the 10 year graft survival rate of $66.4 \%$. What a great success!

I go back to my story. We performed 200 cases of liver transplant surgery at UUH by March 2019. There were 3 cases of retransplantation within 30 days after the primary operation and all of them are alive now. Among 10 cases of 90 -days mortality only 1 patient was succumbed to the surgical cause, portal vein thrombosis. Long-term survival rates were $92.4 \%, 86.8 \%$, and $83.7 \%$ at 1 year, 5 years and 10 years after LT, respectively. The 10 years survival rate for patient with HBV without HCC was $94.4 \%$. I believe these results would have given faith to the local people.

By applying the surgical techniques learned from liver transplantation, more complicated surgery including vascular resection and reconstruction can be performed in the field of hepatobiliary-pancreatic surgery. Vascular resection +/- reconstruction including superior mesenteric-portal vein (SMPV), inferior vena cava (IVC) and/or artery was performed for oncologic reason in 68 cases (22.3\%) among 304 cases of pancreatoduodenectomy (PD) or total pancreatectomy during the period between 2005 and 2020 . The rate of concomitant vascular resection among 76 pancretic ductal adenocarcinoma (PDAC) patients (excluding patients with adenocarcinoma arising from intraductal papillary mucinous neoplasm or neuroendocrine cancer) who underwent PD was very high, $57.9 \%$ (44 patients). The vascular resection included SMPV, IVC, hepatic artery or even superior mesenteric artery (SMA). Various reconstruction methods were applied after SMPV resection, including lateral excision with longitudinal or transverse closure, segmental resection 
with primary end-to-end anastomosis or graft interposition using the autologous left renal vein or cryopreserved allogeneic iliac vein. These 76 PDAC patients had survival rates of $24.2 \%$ and $17.3 \%$, respectively, at 5 and 10 years postoperatively. This figure seems comparable to the result of Japan Pancreatic Cancer Registry, 18.8\% 5 year survival rate after resection. 5 year survival rate for those 44 patients who underwent concomitant vascular resection was $26.2 \%$ and was not different significantly from $21.7 \%$ of the patients who did not undergo vascular resection $(p=0.725)$. Notably, 3 patients underwent concomitant SMA resection and reconstruction and one patient is alive for 4 years and 5 months without PDAC recurrence. SMPV resection and reconstruction following left-sided pancreatectomy is more demanding than after PD. We performed SMPV resection and reconstruction with cryopreserved allogeneic iliac vein following distal pancreatectomy with celiac artery resection (DP-CAR) as a conversion surgery after neoadjuvant chemotherapy for pancreatic adenocarcinoma successfully.

Minimally invasive approach is in the spotlight nowadays in every field of HBP surgery including hepatectomy. Laparoscopic liver resection (LLR) was begun in 2008 and robotic hepatectomy in 2019 at UUH. 174 (25\%) cases out of 688 hepatectomies were performed laparoscopically during the period from August 2008 to November 2020 at UUH. Cases of gallbladder cancer and perihilar cholangiocarcinoma which possibly need biliary manipulation in addition to hepatectomy were excluded. At the beginning, LLR was naturally performed for tumors located at easy anterolateral location. Proportion of LLR is ever increasing, and reached 68\% (40/59) in 2020. Among them are high-level operations such as posterior sectionectomy, central bisectionectomy, anterior sectionectomy and S5 monosegmentectomy under Glisson pedicle approach. 5 year survival rates after LLR were 79.4\% for HCC and 80\% for colorectal cancer liver metastasis (CRLM).

HCC and CRLM are notorious for their tendency of intrahepatic recurrence. 5 year recurrence rates were $46.5 \%$ for HCC and $70.4 \%$ for CRLM at UUH. Repeat liver resection (RLR) is one of the strongest modality for local control of the recurrent tumors. However, RLR is often a very demanding operation due to tough adhesion and distortion of the anatomy. The presence of portal hypertension adds to the difficulty. Among the previous 688 cases, 49 operations were performed for recurrent liver diseases. One patient underwent hepatectomy 4 times and 6 patients 3 times. They were counted each. For 33 HCC patients, 5 and 10 year survival rates after repeat hepatectomy were $66.0 \%$ and $52.8 \%$, respectively. I think it deserves to undergo a RLR for recurrent HCC. Laparoscopic repeat liver resection (LRLR) was begun in 2015 at UUH and accounts for 48\% (14/29) of RLR since then. LRLR group $(n=14)$ was compared to open RLR group (ORLR group, $\mathrm{n}=35)$. There was a considerable trend to perform RLR by laparotomy for larger tumor $(p=0.055)$. ORLR was chosen for tumors in posterosuperior region or bilateral region more frequently $(p=0.032)$. However, RLR can be done by laparoscopic approach safely and with shorter operative time (260 minutes and 336 minutes, $p=0.014$ ), smaller amount of intraoperative blood loss (371 $\mathrm{g}$ and $900 \mathrm{~g}, p=0.028)$ and shorter length of hospital stay ( 7.4 days and 17.5 days, $p=0.005)$ than by open approach in those selected patients.

My story went back and forth a little, from liver transplant to the pancreatectomy and then to the hepatectomy until now. The point I want to talk to the young HBP surgeons today is about the general specialist of HBP surgery. The situation of HBP surgeons working at a local province hospital is quite different from that of HBP surgeons working at the big hospitals in capital area where they usually got fellowship training. For HBP surgeons working at a local province hospital there is almost no choice for sub-sub division like pancreas-only, liver-only or liver transplant-only. She or he must be a general specialist of HBP surgery who is competent in every HBP fields, that is, hepatic surgery, biliary surgery, pancreatic surgery as well as liver transplantation surgery.

How can you make it? I think it is possible by self-directed learning. In these days, there are a lot of platforms guiding you to the cutting-edge knowledge. High-quality knowledge can be acquired through the medical conferences like this HBP Surgery Week. I recommend you to participate in the academic society as actively as possible and also in socializing as well because if you go together, you can go not only further but also faster actually. And you can learn a lot of new knowledge including surgical techniques with video not only by electronic journals but also through the internet like Youtube or IRCAD. Through this self-directed learning, you can overcome the shortage of experience. What is needed is your enthusiasm. As your knowledge and experience increase, the local people will trust you and become willing to have an operation from you. It is mutually beneficial to you and your local people. I believe all the HBP surgeons are so eager or Hagozibi in Korean language that all of you can make it.

Thank you for reading. 